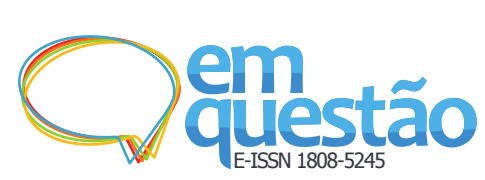

\title{
Rede de relações entre institutos federais de educação e as universidades públicas do sul do Brasil
}

\author{
Renata Ivone Garcia \\ Mestre; Instituto Federal de Santa Catarina, Araranguá, SC, Brasil \\ renataivgarcia@gmail.com \\ Márcio Matias \\ Doutor; Universidade Federal de Santa Catarina, Florianópolis, SC, Brasil; \\ Matias97@gmail.com
}

\begin{abstract}
Resumo: O estudo teve por objetivo analisar, por meio de técnicas e métricas de Análise de Redes Sociais, a rede de conexões estabelecidas, por menções web, entre os Institutos Federais de Educação, Ciência e Tecnologia da região sul do Brasil e as universidades públicas da mesma região. O estudo caracterizou-se como descritivo, e apresentou abordagem quali-quantitativa por meio do emprego de métricas de Análise de Redes Sociais e categorização de conteúdo. Dos resultados de coleta, foram identificadas 25.507 menções web válidas entre as Instituições de Ensino Superior. O Instituto Federal de Educação, Ciência e Tecnologia com maior centralidade de grau foi o Instituto Federal de Santa Catarina. No que diz respeito à densidade, foi percebido que todas as Instituições de Ensino Superior possuem menções web entre si, representando assim uma rede altamente conectada. Mas, foram percebidos três clusters que estabeleceram um número maior de conexões, que são justamente os relacionados a cada estado do sul do Brasil, o que evidencia o fato de que instituições, geograficamente mais próximas, tendem a estabelecer laços fortes. Nos laços fortes, foram identificados os cinco contextos de conteúdo mais recorrentes: eventos científicos; elementos de estudo; divulgação geral; periódicos científicos e qualificação de servidores. Concluiu-se, que estudos envolvendo as menções web e a Análise de Redes Sociais podem ser empregados para estudar as relações entre Institutos Federais de Educação, Ciência e Tecnologia e universidades, o que permitirá, em estudos futuros, focar em aspectos específicos dessas relações, como a cooperação na produção e publicação de artigos científicos.
\end{abstract}

Palavras-chave: Webometria. Menção web. Análise de Redes Sociais. Instituto Federal de Educação, Ciência e Tecnologia. 


\section{Introdução}

$\mathrm{Na}$ sociedade atual, o ambiente web, além de apresentar um crescimento exponencial nos últimos anos em termos de dimensão, tem se caracterizado como um espaço cada vez mais representativo para a disseminação de informação, bem como de comunicação entre indivíduos e entre organizações.

O avanço da tecnologia e a expansão da web têm alterado de maneira significativa as formas de produção, consumo e disseminação da informação, de maneira que é preciso desenvolver estudos que possam medir seus efeitos (ARAÚJO, 2015). Nesse sentido, pesquisas abordando o aspecto da comunicação existente no espaço web, bem como das informações disseminadas, estão sendo desenvolvidas por diferentes áreas do conhecimento, especialmente no âmbito da Ciência da Informação (CI). De acordo com Le Coadic (2004, p. 25), a CI “[...] tem por objeto o estudo das propriedades gerais da informação (natureza, gêneses, efeitos), e a análise de seus processos de construção, comunicação e uso.”. Dessa maneira, dentro da CI, entre as pesquisas e técnicas existentes para analisar os processos de construção, comunicação e uso da informação no ambiente web, estão aquelas relacionadas à webometria.

Segundo Björneborn (2004, p. 12, tradução nossa), a webometria é “[...] o estudo dos aspectos quantitativos da construção e utilização dos recursos informacionais, estruturas e tecnologias na web, utilizando enfoques bibliométricos e infométricos.". E tem por objetivo "Avaliar o sucesso de determinados sítios, detectar a presença de países, instituições e pesquisadores na rede e melhorar a busca e eficiência dos motores de busca na recuperação das informações.” (VANTI, 2002, p. 160). Por meio das técnicas, métodos e indicadores da webometria, é possível avaliar a relevância de instituições, inclusive a de Instituições de Ensino Superior (IES), comumente chamados de websites acadêmicos.

Para Thelwall e Sud (2011, p. 1489), a aferição de links entre websites acadêmicos pode ser considerada indicador para a pesquisa, mais que isso, esses dados medem também “[...] a extensão da sua publicação na web, seu tamanho, sua fama, a fama de seus pesquisadores, atividades profissionais e sua 


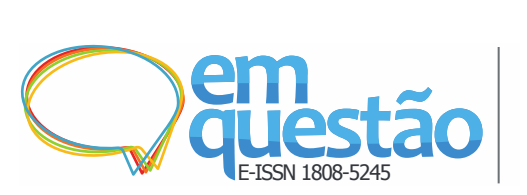

\section{Rede de relação entre institutos federais de} educação e as universidades públicas do sul do Brasil

Renata Ivone Garcia e Márcio Matias

contribuição à educação.”. Destaca-se que, por serem organizações complexas, com websites compostos por diversas páginas web que dão suporte à comunicação científica, as IES tornaram-se principais objetos de estudo dentro da webometria.

Ressalta-se que grande parte das pesquisas desenvolvidas nessa área baseia-se no estudo das conexões entre websites por meio de links, no entanto também estão sendo desenvolvidas investigações que abordam as menções web, que não estão necessariamente atreladas ao link e sim a qualquer parte da página $w e b$. As menções web, entre outros elementos, vêm a contribuir para mensurar e documentar a influência de determinada instituição dentro da rede. Ademais, auxilia no estudo das conexões entre redes específicas da web, com o intuito de mapear as organizações e conexões existentes entre elas (SILVEIRA, 2016).

As menções web dizem respeito "[...] ao número de vezes que o título de um documento, o nome de uma instituição, tópico, objeto ou pessoa aparece nos resultados de uma consulta de mecanismo de pesquisa." (ORTEGA; ORDUÑAMALEA; AGUILLO, 2014, p. 61, tradução nossa). Nesse sentido, as menções web surgem como alternativa aos links, que nos últimos anos vem passando por restrições nos mecanismos de busca de forma gratuita. Por meio dessa técnica, que possui como principal benefício a disponibilidade em motores de busca comercial, é possível identificar a visibilidade web de determinadas instituições, ou, até mesmo, identificar redes de relacionamentos web entre organizações, documentos, teorias e pessoas, por exemplo.

Entre as organizações brasileiras que ofertam cursos em nível de ensino superior e que estão presentes no ambiente web, encontram-se os Institutos Federais de Educação, Ciência e Tecnologia (IF), que se configuram como instituições híbridas, ofertando cursos em diferentes modalidades de ensino. De acordo com o Art. $2^{\circ}$ da Lei 11.892 de 29 de dezembro de 2008, os institutos federais são “[...] instituições de educação superior, básica e profissional, pluricurriculares e multicampi, especializados na oferta de educação profissional e tecnológica nas diferentes modalidades de ensino [...]" (BRASIL, 2008).

Apesar de terem sido criados recentemente com essa nomenclatura e estrutura, os IF são instituições que, em considerável número, migraram das chamadas Escolas de Aprendizes Artífices, instituídas por meio do decreto $n^{\circ}$ 
Rede de relação entre institutos federais de educação e as universidades públicas do sul do Brasil

Renata Ivone Garcia e Márcio Matias

7.566, de 23 de setembro de 1909 (BRASIL, 1909). Essas Escolas passaram, ao longo do tempo, por diferentes mudanças e alterações de status, desde Escola Técnica Federal (ETF) a Centro Federal de Educação Tecnológica (CEFET), que foi a última nomenclatura utilizada antes da migração para IF.

Acredita-se que, assim como já ocorre de maneira acentuada entre as universidades do país, exista, atualmente, um fluxo de informações e cooperação entre os IF e as universidades públicas brasileiras. Essas conexões podem ocorrer por meio da movimentação de colaboradores em busca de capacitação profissional, parcerias em projetos de pesquisa e extensão, envolvimento da instituição ou mesmo dos colaboradores em eventos científicos, cooperação no desenvolvimento de programas de ensino, e até mesmo na participação de colaboradores em bancas de defesa de trabalhos acadêmicos.

As conexões supracitadas dão origem a uma rede de relacionamentos entre IF e universidades públicas do país, de maneira a serem estabelecidas alianças e parcerias, e difundidas ações e conceitos. Nesse contexto de redes, tem-se comumente a aplicação da chamada Análise de Redes Sociais (ARS), que apresenta, como suporte de pesquisa, as conexões estabelecidas dentro de uma rede de atores. Para Bento (2015), a ARS foca nos relacionamentos e nos cenários nos quais eles se constroem, e ainda busca analisar os fluxos de informação com o objetivo de estabelecer categorias qualitativamente adequadas e relevantes.

Estudos quali-quantitativos de menções web entre instituições e/ou organizações, em geral, são escassos (SILVEIRA; MATIAS, 2018), e estudos de menções web entre IF, ou ensino profissional e tecnológico e universidades, não foram encontrados na literatura indexada nas bases de dados Web of Science e Scopus.

Este trabalho tem por objetivo analisar, por meio de técnicas e métricas de ARS, a rede de conexões existentes, por menções web, entre os IF da região sul do Brasil e as universidades públicas da mesma região. Dessa forma, esta pesquisa traz contribuições inéditas para a identificação e a compreensão de conexões existentes entre institutos de educação profissional e tecnológica, e universidades públicas de uma região, registradas na forma de menções web em seus websites. 


\section{Metodologia}

Este estudo utilizou uma abordagem quali-quantitativa. Assim, foram coletadas as quantidades de menções web entre as IES estudadas por meio de técnicas webométricas e em seguida, para a análise quantitativa dos dados, foram empregadas métricas de ARS, focando os padrões de relacionamento estabelecidos na rede formada entre as IES; e, buscando aprofundar a análise dessas relações, foi realizada uma categorização dos contextos de criação das menções web. A natureza quantitativa desse método é evidenciada por Tomaél (2005, p. 138) quando afirma que "A ARS constitui-se em uma metodologia quantitativa de pesquisa que visa à identificação da estrutura de comunicação de um sistema, analisando-a por meio de suas relações.”.

Ademais, “[...] trata-se de um estudo e da descrição das características, propriedades ou relações existentes na comunidade, grupo ou realidade pesquisada." (CERVO; BERVIAN; SILVA, 2013, p. 62). Dessa maneira, o estudo dispõe-se, ainda, a aferir, por meio de menções web, as relações existentes entre os IF da região sul do Brasil e as universidades públicas da região sul brasileira, de maneira a efetuar categorização dos contextos de criação de menções web.

Para identificar os IF existentes na região sul do Brasil, foi realizada busca, em 2 de junho de 2017, no cadastro e-MEC de Instituições e Cursos de Educação Superior ${ }^{1}$, mantido pelo Ministério da Educação (MEC). Após a conferência dos resultados, foram observados seis IF que compõem a região sul brasileira.

Para definir o corpus da pesquisa, buscaram-se as universidades públicas da região sul do país que possuem maior representatividade na web. Dessa maneira, foi utilizada como referência, a edição de janeiro do ano de 2017 do Ranking Webometrics das Universidades do Mundo, que é uma iniciativa do Cybermetrics Lab, localizado na cidade de Madri na Espanha. Esse ranking é construído a partir de dados da web publicamente disponíveis, combinado a outras variáveis em um indicador composto, e com uma verdadeira cobertura global (WEBMETRICS, 2017). Nesse sentido, foram identificadas as dez 


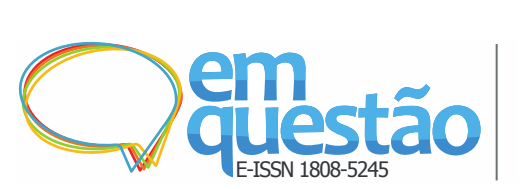

\section{Rede de relação entre institutos federais de educação e as universidades públicas do sul do Brasil \\ Renata Ivone Garcia e Márcio Matias}

universidades públicas da região sul do Brasil que possuem melhor colocação no Ranking Webometrics.

Por fim, o corpus total da pesquisa foi formado por 16 IES, das quais seis são IF da região sul e dez são universidades públicas da região sul, sendo estes últimos com as melhores colocações no Ranking Webometrics. No Quadro 1, podem ser visualizados os dados das IES que compõem o corpus da pesquisa.

Quadro 1 - IES que formam o corpus da pesquisa

\begin{tabular}{|c|l|c|c|}
\hline Estado & \multicolumn{1}{|c|}{ Nome da IES } & Sigla da IES & Website \\
\hline \multirow{2}{*}{ SC } & $\begin{array}{l}\text { Instituto Federal de Educação, Ciência e } \\
\text { Tecnologia de Santa Catarina }\end{array}$ & IFSC & www.ifsc.edu.br \\
\hline SC & $\begin{array}{l}\text { Instituto Federal de Educação, Ciência e } \\
\text { Tecnologia Catarinense }\end{array}$ & IFC & www.ifc.edu.br \\
\hline \multirow{2}{*}{ PR } & $\begin{array}{l}\text { Instituto Federal de Educação, Ciência e } \\
\text { Tecnologia do Paraná }\end{array}$ & IFPR & www.ifpr.edu.br \\
\hline \multirow{2}{*}{ RS } & $\begin{array}{l}\text { Instituto Federal de Educação, Ciência e } \\
\text { Tecnologia do Rio Grande do Sul }\end{array}$ & IFRS & www.ifrs.edu.br \\
\hline \multirow{2}{*}{ RS } & $\begin{array}{l}\text { Instituto Federal de Educação, Ciência e } \\
\text { Tecnologia Sul-Rio-Grandense }\end{array}$ & IFSul & www.ifsul.edu.br \\
\hline RS & $\begin{array}{l}\text { Instituto Federal de Educação, Ciência e } \\
\text { Tecnologia Farroupilha }\end{array}$ & IFFarroupilha & www.iffarroupilha.edu.br \\
\hline SC & Universidade do Estado de Santa Catarina & UDESC & www.udesc.br \\
\hline SC & Universidade Federal de Santa Catarina & UFSC & www.ufsc.br \\
\hline PR & Universidade Estadual de Londrina & UEL & www.uel.br \\
\hline PR & Universidade Estadual de Maringá & UEM & www.uem.br \\
\hline PR & Universidade Federal do Paraná & UFPR & www.ufpr.br \\
\hline PR & Universidade Tecnológica Federal do Paraná & UTFPR & www.utfpr.edu.br \\
\hline RS & Universidade Federal do Rio Grande & FURG & www.furg.br \\
\hline RS & Universidade Federal do Rio Grande do Sul & UFRGS & www.ufrgs.br \\
\hline RS & Universidade Federal de Pelotas & UFPEL & www.ufpel.edu.br \\
\hline RS & Universidade Federal de Santa Maria & UFSM & www.ufsm.br \\
\hline
\end{tabular}

Fonte: Dados da pesquisa.

Para aferir, por meio de menções web, as conexões existentes entre os IF do sul do Brasil, e entre eles e as universidades públicas da região sul do Brasil, foi utilizada uma matriz $\mathrm{N}$ x $\mathrm{N}$ de conexões ou adjacente ator-ator.

Para a coleta de dados da formação dessa rede de conexões, foram utilizados os recursos do motor de busca Google, tendo em vista a característica de popularidade. O Google é o website mais acessado no Brasil e no mundo, levando em consideração a lista divulgada pela Alexa, companhia que fornece informações e análises sobre fluxos de dados na internet (AGRELA, 2017).

No motor de busca Google foi feito uso de um comando de busca específico para recuperar menções web entre duas IES, repetindo-o para cada 


\section{C $\begin{aligned} & \text { Rede de relação entre institutos federais de } \\ & \text { educação e as universidades públicas do sul do }\end{aligned}$ Brasil \\ Renata Ivone Garcia e Márcio Matias}

par de IES que compõe o corpus da pesquisa. Este comando, bem como sua definição, pode ser visualizado no Quadro 2.

Quadro 2 - Comando para mensuração de menção web

\begin{tabular}{|c|c|}
\hline Comando & Definição \\
\hline site:xxxx.edu.br "nome da outra IES" & $\begin{array}{c}\text { Mensura a quantidade de páginas do website X } \\
\text { que mencionam o nome da outra IES }\end{array}$ \\
\hline
\end{tabular}

Fonte: Dados da pesquisa.

A coleta de dados para formação da rede de conexões entre as IES, representada pelo grafo, foi conduzida de maneira sociocentrada, ou seja, buscando compreender a estrutura de conexões de um grupo específico, que neste estudo é representada pelas IES e seus websites. O grafo é do tipo direcionado, onde é possível visualizar os sentidos das menções web e não somente a evidência de alguma conexão ou não entre pares de IES. Com esse tipo de grafo, gerado por uma matriz assimétrica, ou seja, direcionada, é possível evidenciar as possíveis influências de uma IES sobre a outra. Nesse sentido, as menções web foram ponderadas, de maneira que, para cada conexão (aresta), foi determinado um peso que corresponde ao número de menções web detectadas.

O grafo da rede formado de conexões entre as IES foi elaborado por meio do software Gephi, versão 0.9.1, que é uma plataforma de código aberto para a visualização e manipulação de grafos dinâmicos e hierárquicos, incluindo todos os tipos de redes e sistemas complexos (MARQUEZ et al., 2013).

Além da distribuição das menções web (arestas) e das IES (vértices ou nós) no grafo formado, o Gephi proporciona uma avaliação de várias métricas, o que permitiu obter um maior entendimento da rede como um todo e dos vértices que a compõem. Assim, por meio do software, foram mensuradas as seguintes métricas da ARS: tamanho da rede; ordem da rede; diâmetro da rede; densidade da rede; modularidade; centralidade de grau e laços fortes.

$\mathrm{O}$ entendimento inicial era trabalhar somente com as siglas das instituições, no entanto, após as primeiras buscas, percebeu-se um número expressivo de resultados equivocados por conta da ambiguidade das siglas dos IF. Junto a este aspecto, buscou-se recuperar o maior número de menções web entre as IES, optando-se, por fim, adotar variações dos nomes dos IF e universidades, pois tais instituições são conhecidas, com frequência, por 


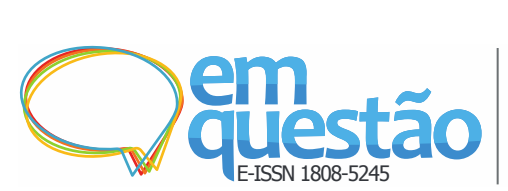

Rede de relação entre institutos federais de educação e as universidades públicas do sul do Brasil

Renata Ivone Garcia e Márcio Matias

diferentes nomenclaturas, alternando entre siglas e nomes por extenso. Para Thelwall, Sud e Wilkinson (2012, p. 808), esse contratempo referente aos diferentes nomes e termos pelos quais uma organização é conhecida “[...] pode ser resolvido usando várias pesquisas, uma para cada variante de texto, eliminando duplicatas e totalizando os resultados restantes.". Esse trabalho manual adicional já é previsto para este tipo de abordagem, pois, como afirmam Thelwall e Sud (2011), a principal desvantagem da menção de título é a necessidade de trabalho manual para identificar as variações dos nomes das organizações, e as combinações eficazes de pesquisas para capturar as formas mais comuns em que uma organização é conhecida na web.

De modo geral, foram adotadas duas variações de nomenclatura para as IES: por sigla e por extenso. No caso de nomes por extenso, foi utilizado o recurso de busca com aspas duplas (“" "), a fim de se recuperar as páginas que continham a sequência exata dos termos existentes nas nomenclaturas da IES.

Thelwall e Sud (2011) relataram que, além da necessidade de identificação das variações de nomenclaturas para cada instituição, é fundamental fazer uso de associações para diminuir a incidência de ambiguidades. Nesse sentido, durante o processo de coleta das menções web, foi identificado, em alguns momentos, a incidência de um número significativo de ruídos relacionados a ambiguidade das nomenclaturas das IES. Assim, visando diminuir distorções nos resultados recuperados, também foi utilizado o recurso sinal de negativo (-) para excluir ambiguidades em algumas estratégias de busca.

Ao total, foram realizados 332 cruzamentos, correspondentes a 150 cenários diferentes de menções web entre pares de IES. Toda a coleta foi realizada entre os meses de novembro e dezembro de 2017, utilizando o buscador Google e o navegador da web Chrome.

Em cada página recuperada durante a coleta, foi verificado se esta, de fato, continha a menção web da IES presente na estratégia adotada, ademais, observou-se se ocorriam ambiguidades com o termo representante da IES. As páginas recuperadas de cada cruzamento realizado foram salvas em planilhas distintas para futuras análises propostas na pesquisa. Tendo em vista a adoção de diferentes nomenclaturas para cada IES, fez-se necessário, primeiramente, a exclusão das páginas duplicadas recuperadas em estratégias de busca distintas. 


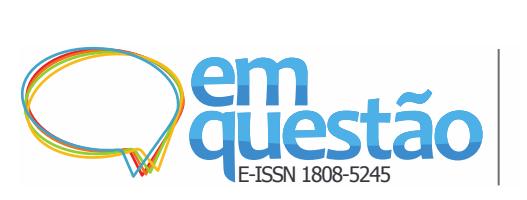

Rede de relação entre institutos federais de educação e as universidades públicas do sul do Brasil

Renata Ivone Garcia e Márcio Matias

Com o intuito de aprofundar a exploração da rede de conexões entre as IES, foi realizada uma análise qualitativa de amostras das conexões mais fortes entre cada IF com outro IF ou com as universidades públicas. A relevância dessa análise qualitativa é evidenciada por Thelwall (2014, p. 31, tradução nossa) quando afirma que "[...] uma análise de conteúdo de uma amostra aleatória dos links é altamente desejável, a fim de ser capaz de interpretar a contagem de links.”, ou seja, a análise de conteúdo busca observar a razão e o contexto da criação da menção web. Nota-se que essa abordagem qualitativa da webometria já era prevista por Thelwall, Vaughan e Björneborn (2005), quando afirmam que a webometria atua em quatro diferentes aspectos de investigação, sendo uma delas a análise de conteúdo das páginas web. Neste caso, buscou-se compreender o contexto da criação das menções web entre duas IES, ou seja, a conjuntura de criação da menção web, a partir da análise qualitativa de conteúdo.

\section{Análise dos resultados}

Após o processo descrito de coleta de dados, foram realizados 332 cruzamentos considerando todas as estratégias adotadas, o que permitiu a identificação de 25.507 menções web válidas entre as IES. Com esses resultados, foi elaborada uma matriz adjacente ator-ator com os 150 diferentes cenários de conexões por menções web entre pares de IES (Quadro 3).

Quadro 3 - Matriz com os resultados das menções web

\begin{tabular}{|c|c|c|c|c|c|c|c|c|c|c|c|c|c|c|c|c|c|}
\hline & $\begin{array}{l}\text { IFS } \\
\text { C } \\
\end{array}$ & $\begin{array}{l}\text { IF } \\
\text { C }\end{array}$ & $\begin{array}{l}\text { IFP } \\
\mathbf{R}\end{array}$ & $\begin{array}{l}\text { IFR } \\
\text { S } \\
\end{array}$ & $\begin{array}{l}\text { IFS } \\
\text { ul }\end{array}$ & $\begin{array}{l}\text { IFF } \\
\text { ar }\end{array}$ & $\begin{array}{l}\text { UFR } \\
\text { GS }\end{array}$ & $\begin{array}{l}\text { UFS } \\
\text { C }\end{array}$ & $\begin{array}{l}\text { UFP } \\
\mathbf{R}\end{array}$ & $\begin{array}{l}\text { UFS } \\
\text { M } \\
\end{array}$ & $\begin{array}{l}\text { UE } \\
\mathbf{L}\end{array}$ & $\begin{array}{l}\text { UE } \\
\text { M } \\
\end{array}$ & $\begin{array}{l}\text { UFP } \\
\text { EL }\end{array}$ & $\begin{array}{l}\text { UTFP } \\
\text { R } \\
\end{array}$ & \begin{tabular}{|l} 
FUR \\
G \\
\end{tabular} & $\begin{array}{l}\text { UDE } \\
\text { SC }\end{array}$ & Total \\
\hline IFSC & - & 390 & 143 & 198 & 112 & 142 & 252 & 649 & 242 & 212 & 109 & 105 & 130 & 180 & 85 & 266 & 3.215 \\
\hline IFC & 229 & - & 100 & 97 & 74 & 158 & 210 & 490 & 174 & 158 & 72 & 61 & 111 & 109 & 37 & 199 & 2.279 \\
\hline IFPR & 160 & 118 & - & 86 & 94 & 123 & 155 & 186 & 370 & 105 & 177 & 218 & 52 & 325 & 53 & 61 & 2.283 \\
\hline IFRS & 200 & 109 & 72 & - & 148 & 366 & 645 & 241 & 129 & 538 & 59 & 54 & 193 & 135 & 208 & 160 & 3.257 \\
\hline IFSul & 88 & 35 & 55 & 141 & - & 163 & 288 & 158 & 102 & 146 & 26 & 29 & 345 & 103 & 174 & 27 & 1.880 \\
\hline IFFar & 176 & 69 & 65 & 170 & 206 & - & 300 & 162 & 85 & 564 & 28 & 32 & 221 & 56 & 217 & 45 & 2.396 \\
\hline $\begin{array}{l}\text { UFRG } \\
\text { S }\end{array}$ & 225 & 180 & 149 & 506 & 533 & 380 & - & - & - & - & - & - & - & - & - & - & 1.973 \\
\hline UFSC & 679 & 600 & 189 & 218 & 194 & 146 & & - & - & - & - & - & & - & - & & 2.026 \\
\hline UFPR & 201 & 127 & 462 & 119 & 90 & 58 & - & - & - & - & - & - & - & - & - & - & 1.057 \\
\hline UFSM & 107 & 135 & 80 & 200 & 162 & 702 & - & - & - & - & - & - & - & - & - & - & 1.386 \\
\hline UEL & 52 & 46 & 168 & 40 & 19 & 21 & - & - & - & - & - & - & - & - & - & - & 346 \\
\hline UEM & 62 & 45 & 231 & 29 & 39 & 24 & & & & & - & - & - & & & - & 430 \\
\hline $\begin{array}{c}\text { UFPE } \\
\text { L }\end{array}$ & 55 & 46 & 13 & 111 & 618 & 91 & - & - & - & - & - & - & - & - & - & - & 934 \\
\hline
\end{tabular}


Rede de relação entre institutos federais de educação e as universidades públicas do sul do Brasil

Renata Ivone Garcia e Márcio Matias

\begin{tabular}{|c|c|c|c|c|c|c|c|c|c|c|c|c|c|c|c|c|c|}
\hline $\begin{array}{c}\text { UTFP } \\
\mathbf{R}\end{array}$ & 145 & 55 & 285 & 67 & 56 & 36 & - & - & - & - & - & - & - & - & - & - & 644 \\
\hline FURG & 29 & 29 & 28 & 188 & 243 & 67 & - & - & - & - & - & - & - & - & - & - & 584 \\
\hline$\underset{\mathrm{C}}{\text { UDES }}$ & 323 & 242 & 53 & 118 & 58 & 33 & - & - & - & - & - & - & - & - & - & - & 827 \\
\hline Total & $\begin{array}{c}2.73 \\
1\end{array}$ & $\begin{array}{l}2.2 \\
26\end{array}$ & $\begin{array}{c}2.09 \\
3\end{array}$ & $\begin{array}{c}2.28 \\
8\end{array}$ & $\begin{array}{c}2.64 \\
6\end{array}$ & 2.510 & 1.850 & 1.876 & 1.102 & 1.723 & 471 & 499 & 1.052 & 908 & 774 & 758 & \\
\hline
\end{tabular}

Fonte: Dados da pesquisa.

Essa matriz de menções web deu origem a uma rede de conexões formadas entre essas IES. Nesse sentido, buscou-se analisar a estrutura dessa rede por meio de aplicação da ARS, que por sua vez "[...] diz respeito à forma das relações entre os atores sociais.” (LEMIEUX; OUIMET, 2012, p. 11).

No âmbito da ARS podem ser empregadas diferentes métricas que auxiliam na análise pretendida no estudo desenvolvido. Para a presente pesquisa, foram aplicadas as seguintes métricas para a rede: 'tamanho da rede', 'ordem da rede', 'diâmetro da rede' e 'densidade da rede', de maneira que os resultados estão dispostos na Tabela 1 .

Tabela 1 - Resultados das métricas de ARS

\begin{tabular}{c|c}
\hline MÉTRICAS & RESULTADOS \\
\hline Tamanho da rede & 25.517 \\
\hline Ordem da rede & 16 \\
\hline Diâmetro da rede & $1^{*}$ \\
\hline Densidade da rede & 1 \\
\hline
\end{tabular}

Fonte: Dados da pesquisa.

Nota: *Considerando que não foram contabilizadas as menções web entre universidades.

O 'tamanho da rede' diz sobre o número total de menções web identificadas entre as IES por meio de seus websites e a 'ordem da rede' é o número de atores, nesse caso de IES. De acordo com Gabardo (2015), não é possível determinar se o tamanho da rede é considerado 'grande' ou 'pequeno', por exemplo, pois as redes estudadas podem apresentar distintas dimensões de acordo com a proposta do estudo.

Por apresentar densidade um, ou seja, por ser uma rede altamente conectada, onde todas as IES possuem conexões entre si por meio de menções web, o diâmetro da rede também refletiu essa alta densidade apresentada pela rede estudada. Pelo fato de terem sido identificadas conexões entre todas as IES, a distância geodésica entre todas as IES não ultrapassou um ponto. No que diz respeito à densidade da rede, Recuero (2017) afirma que tal métrica reflete a 


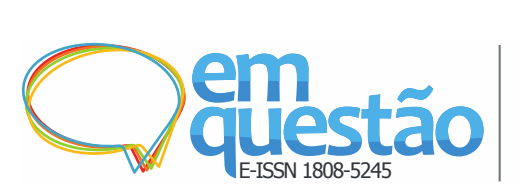

Rede de relação entre institutos federais de educação e as universidades públicas do sul do Brasil

Renata Ivone Garcia e Márcio Matias

existência de conexões para todos pares de IES, ou seja, a densidade demonstra o quão conectada uma rede está.

A 'densidade da rede' e a 'ordem da rede' podem estar intimamente relacionadas entre si, pois, de acordo com Lemieux e Ouimet (2012), essas duas métricas são inversamente proporcionais, ou seja, quanto mais atores existirem numa rede menor será a densidade de conexões entre eles. Este entendimento pode ser visualizado na rede estudada, a qual é composta, relativamente, de poucas IES (16), e apresentou um alto índice de conexão, ou seja, densidade.

Apesar do alto índice de densidade na rede, onde todas as IES estão conectadas entre si por meio de menções web, foram identificadas, por meio do software Gephi, três comunidades no que diz respeito à modularidade. A questão geográfica influenciou no peso das conexões entre pares de IES, ou seja, no número de menções web estabelecidas, de maneira que as três comunidades são compostas pelas IES de cada estado do sul do Brasil: Paraná, Rio Grande do Sul e Santa Catarina. No Grafo 1, é possível visualizar a rede de menções web distribuída pelo indicador modularidade.

Grafo 1 - Rede distribuída por modularidade

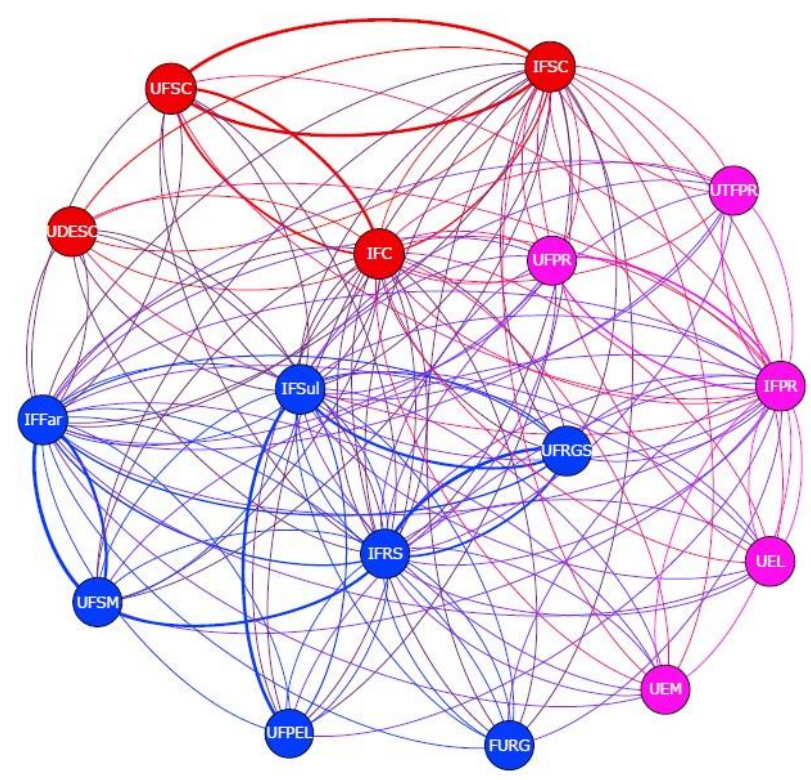

Fonte: Dados da pesquisa.

No Grafo 1, é possível notar três comunidades: nós azuis são IES do Rio Grande do Sul; nós rosa do estado do Paraná e, nós vermelhos constituem-se em IES de Santa Catarina. Essas comunidades, conhecidas também como sub-redes ou clusters, representam um alto grau de similaridade entre os nós, que pode ser 


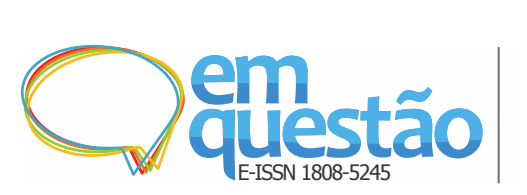

Rede de relação entre institutos federais de educação e as universidades públicas do sul do Brasil

Renata Ivone Garcia e Márcio Matias

identificado pelo número dos pesos das conexões, ou seja, pelas menções web. De acordo com Recuero (2017) em termos sociológicos, a estrutura das comunidades demonstra nós que são mais conectados na rede e que, assim, possuem um maior número de interações.

Além das métricas voltadas à rede, apresentadas anteriormente, foram utilizadas algumas métricas aplicadas aos nós, ou seja, a cada IES que compõe a rede de menções web. A primeira utilizada é a centralidade do grau (degree centrality), que identifica o quão central, ou seja, qual IES possui um maior número de conexões diretas com as outras IES da rede. Como a rede é um grafo direcionado, onde há menção web nos dois sentidos e com conexões valoradas por conta do peso de cada menção, divide-se a centralidade em Indegree e OutDegree.

Comparando-os aos indicadores webométricos, o grau de Indegree é congênere ao indicador 'visibilidade', e o grau OutDegree é análogo ao indicador 'luminosidade'. Nesse sentido, a centralidade Indegree indica popularidade ou receptividade da IES dentro da rede analisada, ao passo que a centralidade OutDegree manifesta expansividade ou poder de prover conexões (LOIOLA; BASTOS; REGIS, 2015). Dessa forma, foi identificado que o IF com maior grau de centralidade Indegree é o Instituto Federal de Santa Catarina (IFSC), pois foi o mais mencionado pelas demais IES da rede. Já o IF que possui maior grau de centralidade OutDegree é o IFRS, ou seja, o seu domínio web (ifrs.edu.br) foi o que mais promoveu menções web na rede.

Quando somadas as centralidades Indegree e OutDegree, tem-se o número de menções web totais relacionadas a cada IF, expondo, assim, a centralidade de grau (degree centrality) total (Tabela 2).

Tabela 2 - Centralidade de grau (degree centrality)

\begin{tabular}{c|c}
\hline IF & $\mathbf{N}^{\mathbf{0}}$ menções $\boldsymbol{w e b}$ \\
\hline IFSC & 5.946 \\
\hline IFRS & 5.545 \\
\hline IFFar & 4.906 \\
\hline IFPR & 4.676 \\
\hline IFSul & 4.526 \\
\hline IFC & 4.505 \\
\hline \multicolumn{2}{c}{ Fonte: Dados da pesquisa. }
\end{tabular}

O IFSC é o nó mais central da rede estudada, ou seja, é a instituição que mais possui menções web, somando, no sentido de mencionado e proprietário do 


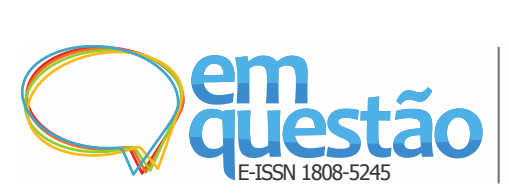

Rede de relação entre institutos federais de educação e as universidades públicas do sul do Brasil

Renata Ivone Garcia e Márcio Matias

domínio, 5.946 menções web. Em seguida, o IFRS é o segundo nó mais central, com 5.545 menções web em suas conexões. O IFFar, com 4.906 menções web, é o terceiro nó que mais apresentou grau de centralização. Seguindo, o quarto nó com maior centralidade é o IFPR apresentando 4.676 menções web em suas conexões com outras IES. Por fim, em quinto e sexto lugares estão o IFSul e IFC, respectivamente, com 4.526 e 4.505 menções web, na mesma ordem.

É relevante mencionar que, quando se foca especificamente as relações estabelecidas diretamente entre os IF e as universidades, percebe-se que, na maioria dos casos, os IF mencionam mais as universidades do que o sentido oposto. Dos 60 cruzamentos estabelecidos entre essas IES, os IF mencionaram mais as universidades em 46 cenários e as universidades mencionaram mais os IF em 14 outros cenários, entre pares dessas IES. Essas menções recebidas (Indegree) pelas universidades, obtidas nas relações estabelecidas com os IF, são compatíveis com a maior visibilidade destas universidades no Ranking Webometrics.

Buscou-se identificar as conexões mais representativas de cada IF em termos de peso, ou seja, dentro do cenário de cada IF, qual a conexão que mais apresentou menções web, representando laços fortes. No Grafo 2, é possível visualizar as arestas (conexões) com maiores pesos dentro da rede de menções web.

Grafo 2 - Rede distribuída pelos pesos das menções web

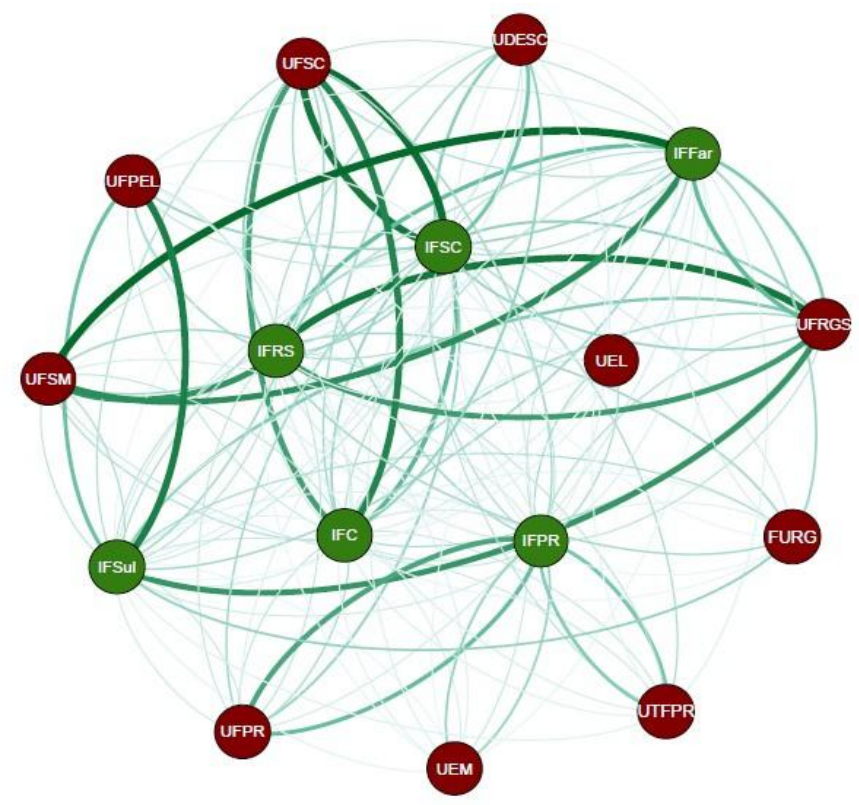

Fonte: Dados da pesquisa. 

educação e as universidades públicas do sul do Brasil

Renata Ivone Garcia e Márcio Matias

No Grafo 2, é possível notar arestas com bordas mais largas e com tons de cores mais escuras, representando assim as conexões com maiores pesos, ou seja, com um número significativo de menções web. As conexões com maiores pesos dos IF, logo com laços mais fortes, são com as universidades federais do mesmo estado. Nesse sentido, percebemos laços fortes em termos de número de menção web nas seguintes conexões dos IF: o IFSul com a UFPel e UFRGS; IFRS com a UFRGS e UFSM; IFFar com a UFSM; IFSC com a UFSC; IFC com a UFSC e, IFPR com a UFPR.

Ao observarmos o Grafos 1 e 2, percebemos que as conexões mais fortes estão presentes entre pares de IES de uma mesma comunidade, o que, de fato, ratifica a afirmação da autora supracitada, de que laços mais fortes estão mais presentes em comunidades identificadas na rede.

Para Recuero (2017), os pesos atribuídos às conexões, neste caso o número de menções web, podem dar pistas do tipo de conexão estabelecida entre os atores da rede estudada. Nessa perspectiva, as conexões mais fracas ou mais fortes, com pesos menores e maiores respectivamente, podem demonstrar o tipo de laço social que existe entre um determinado par de atores. Ademais, a autora afirma que onde há conexões mais fortes, há também conexões mais diversas, provenientes de relações sociais mais variadas. Ainda sobre as conexões mais fortes, estas tendem a ser mais recíprocas e tendem a constituir, na estrutura da rede, comunidades ou clusters.

Buscando expandir o estudo da rede e analisar qualitativamente as conexões mais fortes, ao ponto de identificar quais relações sociais estão ocorrendo entre essas IES, na Figura 1, estão apresentadas as cinco categorias de conteúdo encontradas nesses laços fortes estabelecidos entre os IF e as universidades. 


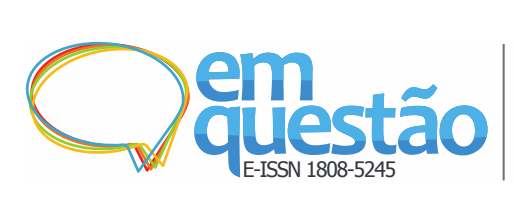

Rede de relação entre institutos federais de educação e as universidades públicas do sul do Brasil

Renata Ivone Garcia e Márcio Matias

Figura 1 - Contexto da menção web - resultados finais

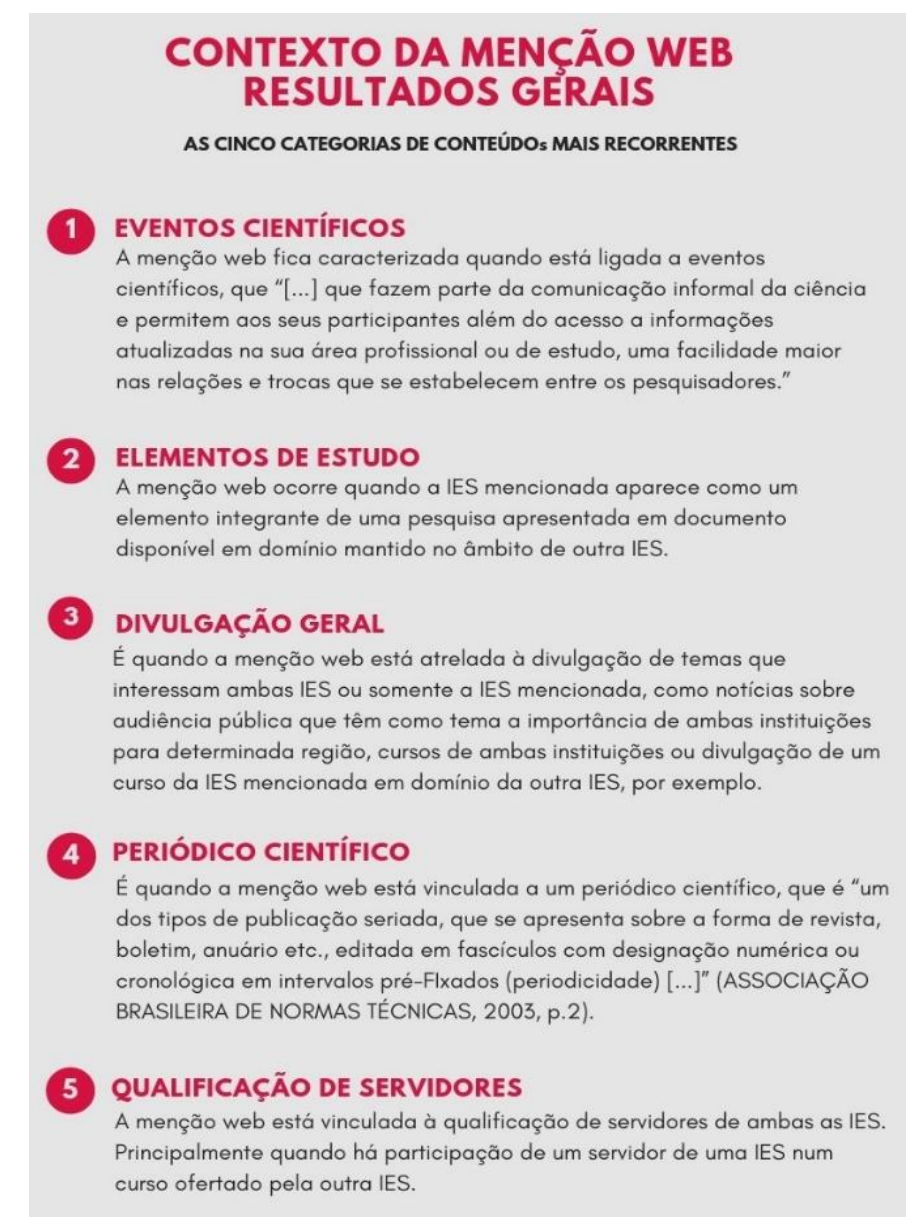

Fonte: Dados da pesquisa.

Pode-se perceber que o contexto da menção web que mais apresentou ocorrências foi o de eventos científicos, de maneira que ficaram evidenciados, entre outros aspectos: a organização de eventos científicos em parceria entre os IF e as universidades; a participação de integrantes da comunidade acadêmica de uma IES como autores de trabalhos científicos apresentados em eventos organizados por outra IES; bem como a ocorrência de palestrante da IES mencionada em eventos organizados pela IES proprietária do domínio.

Além de estarem equiparados com as universidades no que diz respeito à regulação, avaliação e supervisão das instituições e dos cursos de educação superior, os IF também desenvolvem ações de ensino, pesquisa e extensão, no entanto, moderados pela integração entre cultura e, principalmente, ciência e tecnologia pela educação profissional e tecnológica (PACHECO, 2011). Nesse sentido, são desenvolvidas investigações científicas no âmbito dos IF, muitas vezes por meio de projetos e/ou estabelecimento de grupos de pesquisa. 


\section{C $\begin{aligned} & \text { Rede de relação entre institutos federais de } \\ & \text { educação e as universidades públicas do sul do }\end{aligned}$ Brasil \\ Renata Ivone Garcia e Márcio Matias}

Ademais, como se percebe no contexto de eventos científicos, há o estabelecimento de parcerias entre pesquisadores e grupos de pesquisas dos IF e universidades para a organização de eventos científicos de interesse para ambas instituições.

O segundo contexto que mais apresentou incidências de menções web é aquele no qual a IES é mencionada como um elemento de estudo. Essa constatação reflete a grande incidência dos IF do sul do Brasil sendo mencionados como um elemento de estudo em documentos disponibilizados em domínios de universidades públicas também da região sul.

Os resultados supracitados aliados à ocorrência significativa de menções web no contexto da qualificação de servidores dos IF, também contribuem para evidenciar o processo de expansão pelo qual a Rede Federal de Educação Profissional, Científica e Tecnológica passou a partir do ano de 2003. De acordo com dados disponibilizados pelo MEC, a Rede sofreu, nos últimos anos, uma grande expansão em termos de transformação e criação de novas unidades de ensino. De forma que em 93 anos, de 1909 a 2002, foram construídas 140, até então chamadas, escolas técnicas. Já de 2003 a 2016, ou seja, num espaço temporal de 13 anos, o MEC implantou mais de 500 novas unidades referentes ao plano de expansão da educação profissional, totalizando 644 campi em funcionamento até março de 2016 (BRASIL, 2016). Por conseguinte, com a grande expansão e implementação dos novos campi houve a demanda de provimento de vagas para servidores atuarem nessas unidades educacionais.

$\mathrm{O}$ contexto de menções web relacionado à qualificação de recursos humanos está, em grande parte, relacionado à qualificação de servidores dos IF em cursos de pós-graduação ofertados pelas universidades públicas da região.

A expansão da Rede Federal de Educação Profissional, Científica e Tecnológica proporcionou o aumento no número de servidores dessas instituições, que acabam buscando qualificação profissional em universidades próximas a sua região. Ademais, a expansão e também a criação recente dos IF com a nomenclatura e objetivos atuais, representando, assim, uma nova concepção de instituição de ensino, pode ter contribuído para o alto índice dos estudos relacionados aos IF. Nesse sentido, Pacheco, Pereira e Domingos Sobrinho (2010) explanam que, para se firmar como agentes importantes no 


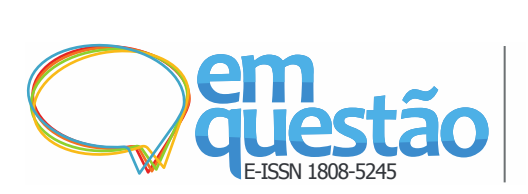

Rede de relação entre institutos federais de educação e as universidades públicas do sul do Brasil

Renata Ivone Garcia e Márcio Matias

desenvolvimento tecnológico nacional, os IF têm, como fatores influenciadores, o aumento da qualificação do quadro de pessoal; a consolidação e a ampliação dos grupos de pesquisa; a articulação com o mundo econômico, particularmente regional e local; e a forte expansão dos campi e as unidades de ensino.

O terceiro contexto de criação de menção web que possui maior número de ocorrências é o de divulgação geral, que não representa filiação entre IF e universidades, mas está atrelado à divulgação de temas que interessam ambas IES ou somente a IES mencionada, como notícias sobre audiência pública que têm como tema a importância de ambas instituições para determinada região, cursos de ambas instituições ou divulgação de um curso da IES mencionada em domínio da outra IES, por exemplo.

Contextos vinculados aos periódicos científicos também foram destaques, e essas menções ocorreram, em maioria, pelo fato de os autores de artigos científicos serem integrantes da comunidade acadêmica da IES mencionada, e também foi percebido colaboração entre integrantes de ambas IES para publicação de trabalhos científicos. Além de um canal importante de disseminação e recuperação da informação, os periódicos científicos possuem um "[...] importante papel de visibilidade, tanto para as publicações, como para seus autores e editores." (FACHIN; HILLESHEIM, 2006, p. 41). Nesse sentido, a relação estabelecida entre os IF e universidades por meio da autoria de artigos científicos, além de promover o pesquisador, que é um integrante da comunidade acadêmica da IES mencionada, dá maior notoriedade à instituição a qual este autor está vinculado.

Após os resultados dessa análise, percebe-se que os tipos de contextos de estabelecimento das conexões mais representativas entre IF e universidades foram os mais diversos, constituindo-se assim em variados laços sociais. De modo que, assim como já ocorre de maneira acentuada entre as universidades do país, existe, de fato, cooperação entre os IF e as universidades públicas do sul do país. E essas conexões ocorrem acentuadamente por meio da movimentação de colaboradores em busca de capacitação profissional e envolvimento da instituição ou mesmo dos colaboradores em eventos científicos. 
Rede de relação entre institutos federais de educação e as universidades públicas do sul do Brasil

Renata Ivone Garcia e Márcio Matias

\section{Considerações finais}

Tendo em vista a relevância dos estudos relacionados à webometria para identificar e compreender as relações estabelecidas entre instituições de ensino por meio da menção web, e considerando-se a natureza singular dos IF no que diz respeito aos seus objetivos, foi estabelecido para esta pesquisa o objetivo de 'Analisar, por meio de técnicas e métricas de ARS, a rede de conexões existentes, por menções web, entre os Institutos Federais de Educação, Ciência e Tecnologia da região sul do Brasil e as universidades públicas da mesma região'.

Para alcançar esse objetivo, foi realizada, primeiramente, uma etapa de trabalho sistemático no que diz respeito à consideração de variações dos nomes dos IF e universidades, pois tais instituições são conhecidas, com frequência, por diferentes nomenclaturas, alternando entre siglas e nomes por extenso. Nessa etapa, realizou-se, portanto, o que é recomendado para se identificar todas as menções web envolvendo as instituições estudadas. Desta forma, entende-se que este recurso de detecção de menções web demonstrou-se eficaz para identificação das relações entre IES registradas na web.

Ao serem identificadas as conexões entre IF e universidades públicas por meio de menções $w e b$, formou-se uma rede de relacionamentos entre essas IES do sul do Brasil. E, para compreender esses laços estabelecidos entre as IES, foram aplicadas métricas de ARS. No que diz respeito à densidade desta rede, foi percebido que todas as IES possuem menções web entre si, representando assim uma rede altamente conectada. No entanto, foram percebidos três subgrupos ou clusters que estabeleceram um número maior de menções, ou seja, possuem conexões mais fortes, que são justamente as IES de cada estado separadamente: Rio Grande do Sul, Santa Catarina e Paraná. Nesse sentido, pode-se reafirmar que instituições congêneres que estão geograficamente mais próximas tendem a apresentar um maior número de conexões e interações sociais.

No que diz respeito às métricas de cada nó, ou seja, relacionadas a cada IES que compõe a rede estudada, destaca-se a centralidade do grau (degree centrality), que busca identificar o nó mais central nesta rede. Por ser uma rede direcionada, foram somadas a centralidade Indegree e a OutDegree, cujo 


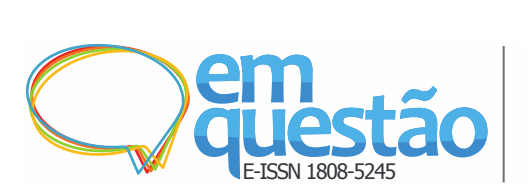

Rede de relação entre institutos federais de educação e as universidades públicas do sul do Brasil

Renata Ivone Garcia e Márcio Matias

resultado identificou o IFSC como a instituição com maior número de menções web.

Relevante considerar que, ao observar estritamente as relações estabelecidas diretamente entre os IF e as universidades, percebeu-se que, em maioria, os IF mencionam mais as universidades do que o sentido oposto. Acredita-se que essa constatação, vai ao encontro da maior visibilidade das Universidades selecionadas para o estudo no Ranking Webometrics.

Tendo em vista que os pesos atribuídos às conexões, que neste estudo correspondem ao número de menções web, podem ser representativos para estabelecer o tipo de conexão, se forte ou fraca, entre duas IES. Foram identificadas as conexões mais fortes relacionadas a cada IF. Seguindo a composição dos clusters supracitados, as conexões com laços mais fortes de cada IF se deram com as universidades federais estabelecidas no mesmo estado.

Observa-se que, por meio da técnica webométrica de menções web, foi possível identificar diferentes contextos de conexões entre IF e universidades públicas da região Sul do Brasil. Esses resultados refletem a relevância das menções web como um instrumento que possibilita identificar e mapear as diversas interações estabelecidas entre instituições de ensino, ou até mesmo de outros tipos de organizações. Essas constatações acerca da menção web ratificam a contribuição desta pesquisa para o campo da webometria, no que diz respeito à relevância da menção de palavras/títulos/termos como uma alternativa viável e eficaz para estudos webométricos.

Entre os contextos de criação de menção web identificados, estão aqueles relacionados a eventos científicos. Nesses resultados, nota-se expressiva interação entre as IES para a organização de eventos em parceria, aspecto relevante para identificação de assuntos pertinentes e comuns entre IF e universidades. São temas de estudo que são de interesse comum entre essas instituições que dão origem à parceria para o desenvolvimento de eventos científicos.

Contextos onde a IES mencionada aparece como um elemento de estudo foram identificados em número representativo no contexto de IF como elementos estudados em pesquisas. Esse resultado, associado ao alto índice de qualificação de servidores, pode estar vinculado ao processo de expansão pelo 


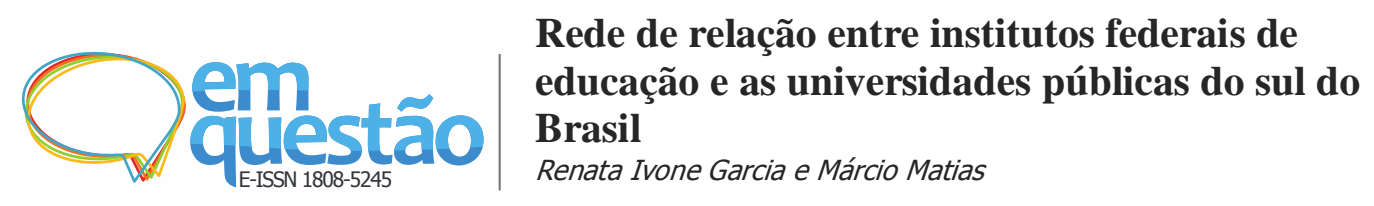

qual a Rede Federal de Educação Profissional, Científica e Tecnológica começou a passar a partir do ano de 2003, com a transformação e a criação de novas unidades de IF.

A técnica webométrica de menções web demonstrou ser eficaz e apropriada para a proposta de pesquisa de analisar as conexões dos IF entre si e com universidades públicas da região sul do Brasil. Ao ponto que uma das contribuiçõoes deste estudo está pautada em que gestores desses IF possam, por meio dessa análise, averiguar como sua instituição está sendo mencionada fora do seu domínio; com qual outra instituição do sul do país estabelece conexões mais fortes; e quais os tipos de laços estabelecidos nessas conexões. De maneira que, munidos desses dados, possam propor ou adequar suas políticas ou estratégias relacionadas a diferentes aspectos, como visibilidades web, estreitamento de parceria de projetos e grupos de pesquisa com outra IES, entre outros.

Ademais, realizando a análise qualitativa das conexões com maiores pesos dentro de cada cenário envolvendo os seis IF, ou seja, as conexões mais fortes, notou-se que, de fato, estas apresentam relações mais diversas, provenientes de laços sociais mais variados.

A aplicação de técnicas e métricas envolvendo a ARS representou recurso relevante para mapear a estrutura da rede criada entre as IES aqui estudadas por meio de menções web. Foi possível identificar e visualizar, por meio de grafos, as posições dos atores na rede, de modo a apontar com qual outra IES há conexões mais fortes, e também clusters, onde há uma troca maior de informações e o estabelecimento de mais cooperação. Esses resultados podem ser potencialmente relevantes para gestores e/ou pesquisadores dessas IES, pois estes podem, além de diagnosticar relações existentes entre as instituições pesquisadas, fundamentar e direcionar políticas de convênios entre essas instituições, por exemplo.

Como sugestões para futuras pesquisas está a exploração da cooperação entre IF de outras regiões do Brasil, em parceria com universidades nacionais e até mesmo, internacionais. Ademais, acredita-se que a ARS, assim como foi empregada nesta pesquisa, possa ser utilizada em estudos pósteros, visto que conforme haja produção em coautoria, com a aplicação da ARS pode-se 


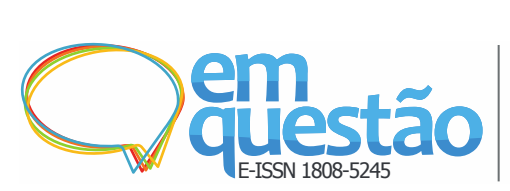

Rede de relação entre institutos federais de educação e as universidades públicas do sul do Brasil

Renata Ivone Garcia e Márcio Matias

identificar os autores com maior número de produções, clusters de cooperação de autores, aspectos geográficos, entre outros.

Por fim, verificou-se que estudos envolvendo as menções web registradas em seus websites podem ser aplicadas sobre as redes de relações entre os tipos de instituições de ensino pesquisadas. Isto leva à possibilidade de exploração, no futuro, por exemplo, de estudos mais específicos sobre a cooperação por meio de composição de banca, publicação de artigos científicos e a organização de eventos científicos.

\section{Referências}

AGRELA, L. Os 50 sites mais acessados do Brasil e do mundo. São Paulo, 2017. Disponível em: http://exame.abril.com.br/tecnologia/os-50-sites-maisacessados-do-brasil-e-do-mundo/. Acesso em: 19 jul. 2017.

ARAÚJO, R. F. de. Os estudos cibermétricos da informação: das estruturas web aos recursos da web social. In: ARAÚJO, R. F. de (Org.). Estudos métricos da informação na $\boldsymbol{w e b}$ : atores, ações e dispositivos informacionais. Maceió:

Edufal, 2015.

BENTO, L. Análise redes sociais virtuais: da estrutura aos relacionamentos. In: GALINDO, D. S. (org.). A comunicação no mercado em redes virtuais: uma questão de relacionamento. Chapecó: Argos, 2015.

BJÖRNEBORN, L. Small-world links structures across an academic web space: a library and information science approach. 2004. 399 f. Thesis (Information Science PhD) - Department of Information Studies, Royal School of Library and Information Science, Dinamarca, 2004.

BRASIL. Decreto no 7.566, de 23 de setembro de 1909. Créa nas capitaes dos Estados da Escolas de Aprendizes Artífices, para o ensino profissional primario e gratuito. Diário Oficial [da] República Federativa do Brasil, Brasília, DF, 26 set. 1909. Seção 1, p. 6975.

BRASIL. Lei n. 11.892, de 29 de dezembro de 2008. Institui a Rede Federal de Educação Profissional, Científica e Tecnológica, cria os Institutos Federais de Educação, Ciência e Tecnologia, e dá outras providências. Diário Oficial [da] República Federativa do Brasil, Brasília, 30 dez. 2008. Seção 1, p. 1.

BRASIL. Portal da Rede Federal de Educação Profissional, Científica e Tecnológica. Brasília, 2016. Disponível em: http://redefederal.mec.gov.br/instituicoes. Acesso em: 15 set. 2017. 


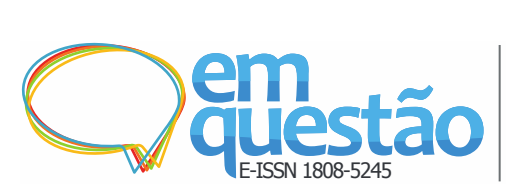

Rede de relação entre institutos federais de educação e as universidades públicas do sul do Brasil

Renata Ivone Garcia e Márcio Matias

BRASIL. Cadastro nacional de cursos e instituições de educação superior. Brasília, 2019. Disponível em: http://emec.mec.gov.br/. Acesso em: 31 mar. 2019.

CERVO, A. L.; BERVIAN, P. A.; SILVA, R. Metodologia científica. 6. ed. São Paulo: Pearson Prentice Hall, 2013.

FACHIN, G. R. B.; HILLESHEIM, A. I. A. Periódico científico: padronização e organização. Florianópolis: UFSC, 2006.

GABARDO, A. C. Análise de redes sociais: uma visão computacional. São Paulo: Novatec, 2015.

LE COADIC, Y. A ciência da informação. 2. ed. Brasília: Briquet de Lemos, 2004.

LEMIEUX, V.; OUIMET, M. Análise estrutural das redes sociais. 2. ed. Lisboa: Instituto Piaget, 2012.

LOIOLA, E.; BASTOS, A. V. B; REGIS, H. P. Análise de Redes Sociais. In: BASTOS, A. V. B; LOIOLA, E.; REGIS, H. P. (org.). Análise das redes sociais em contextos organizacionais. Salvador: EDUFBA, 2015.

MARQUEZ, A. C. et al. Gephi: um software open source de manipulação e visualização de grafos. [S.l.]: Labic, 2013.

ORTEGA, J. L.; ORDUÑA-MALEA, E.; AGUILLO, I. F. Are web mentions accurate substitutes for inlinks for Spanish universities? Online Information Review, [s.l.], v. 38, n. 1, p. 59-77, 2014.

PACHECO, E. Institutos federais: uma revolução na educação profissional e tecnológica. In: PACHECO, E. (Org.). Institutos federais: uma revolução na educação profissional e tecnológica. Brasília: Fundação Santillana, 2011.

PACHECO, E. M.; PEREIRA, L. A. C.; DOMINGOS SOBRINHO, M..

Institutos Federais de Educação, Ciência e Tecnologia: limites e possibilidades. Linhas Críticas, [s.l.], v. 16, n. 30, p. 71-88, jan./jun. 2010.

RECUERO, R. Introdução à análise de redes sociais online. Salvador: Edufba, 2017.

SILVEIRA, E. Conexões entre universidades por meio de citação web: um estudo webométrico nas universidades UFPR, UFRGS e UFSC. 2016. 268 f. Dissertação (Mestrado em Ciência da Informação) - Curso de Programa de PósGraduação em Ciência da Informação, Universidade Federal do Santa Catarina, Florianópolis, 2016.

SILVEIRA, E.; MATIAS, M. Webometria e análise das menções web dos partidos políticos com representação no Senado Federal. Encontros Bibli: 


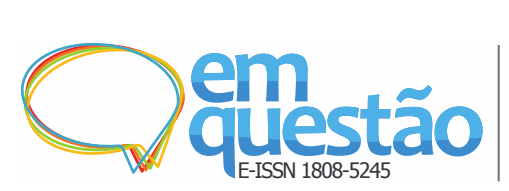

Rede de relação entre institutos federais de educação e as universidades públicas do sul do Brasil

Renata Ivone Garcia e Márcio Matias

revista eletrônica de biblioteconomia e ciência da informação, Florianópolis, v. 23, n. 53, p. 174-183, set./dez., 2018.

THELWALL, M. Big data and social web research methods. 2014.

THELWALL, M.; SUD, P. A comparison of methods for collecting web citation data for academic organizations. Journal of the American Society for Information Science and Technology, New York, v. 62, n. 8, p. 1488-1497, ago. 2011.

THELWALL, M.; SUD, P.; WILKINSON, D. Link and co-inlink network diagrams with URL citations or title mentions. Journal of the American Society for Information Science and Technology, New York, v. 63, n. 4, 805816, 2012.

THELWALL, M.; VAUGHAN, L.; BJÖRNEBORN, L. Webometrics. Anual Review of Information Science and Technology, [s.l.], v. 39, n. 1, p. 81-135, 2005.

TOMAÉL, M. I. Redes de conhecimento: o compartilhamento da Informação e do conhecimento em consórcio de exportação do setor moveleiro. 2005. 292f. Tese (Doutorado em Ciência da Informação) - Universidade Federal de Minas Gerais, Belo Horizonte, 2005.

VANTI, N. Da bibliometria à webometria: uma exploração conceitual dos mecanismos utilizados para medir o registro da informação e a difusão do conhecimento. Ciência da Informação, Brasília, v. 31, n. 2, p. 152-162, maio/ago. 2002.

WEBMETRICS. Methodology. 2017. Disponível em: http://www.webometrics.info/en/Methodology. Acesso em: 23 maio 2017.

\title{
Network of relations between federal institutes of education and the public universities from the south of Brazil
}

\begin{abstract}
The objective of this study was to analyze, through techniques and metrics of Social Network Analysis, the network of connections established by web mentions between the Federal Institutes of Education of the southern region of Brazil and the public universities from the same region. The study was characterized as descriptive and presented a qualitative-quantitative approach through the use of Social Network Analysis metrics and content categorization. The results of the web mentions collection identified 25.507 valid web references among the Institutions of Higher Education. The Federal Institutes of Education with the highest degree centrality was the Federal Institute of Santa Catarina. With regard to density, it was noticed that all Institutions of Higher Education make web mentions among themselves, which represents a highly
\end{abstract}




\section{Rede de relação entre institutos federais de educação e as universidades públicas do sul do Brasil \\ Renata Ivone Garcia e Márcio Matias}

connected network. However, it was perceived three clusters that established a greater number of connections, precisely the Institutions of Higher Education from each state of the southern Brazil, which shows the fact that geographically closer institutions tend to establish strong ties. In the strong ties were identified the five most recurrent content contexts: scientific events; elements of study; general disclosure; scientific journals; and server qualification. It was concluded, therefore, that studies involving web references and Social Network Analysis could be used to study relations between Federal Institutes of Education and universities, which will allow, in future studies, to focus on specific aspects of such relations, such as cooperation in production and publication of scientific articles.

Keywords: Webometrics. Web mention. Social network analysis. Federal Institute of Education, Science and Technology.

Recebido: 22/07/2018

Aceito:16/11/2018

${ }^{1}$ O Cadastro e-MEC de Instituições e Cursos de Educação Superior é base de dados oficial e única de informações relativas às Instituições de Educação Superior - IES e cursos de graduação do Sistema Federal de Ensino (BRASIL, 2019). 